\title{
An approach of classification and parameters estimation, using neural network, for lubricant degradation diagnosis
}

\author{
Gavril Grebenişan ${ }^{1, *}$, Nazzal Salem², and Sanda Bogdan ${ }^{3}$ \\ ${ }^{1}$ University of Oradea, Faculty of Manag. and Technological Eng., Industrial Engineering Department, Universitatii 1, Romania \\ ${ }^{2}$ Zarqa University, Faculty of Engineering Technology, P.O. Box 132222 Zarqa 13132, Jordan \\ ${ }^{3}$ University of Oradea, Faculty of Manag. and Techn. Eng., Mechanical Eng. and Automotive Department, Universitatii 1, Romania
}

\begin{abstract}
This paper addresses a delicate problem, namely the diagnosis of the state of the oils in the industrial systems, namely the machine tools. Based on measurements (the data set contains over five million records), within a Machine Intelligence for Diagnosis Automation (MIDA) project funded by the National Program PN II, ERA MANUNET: NR 13081221 / 13.08.2013, several applications of MATLAB toolbars are being developed in the field of artificial intelligence, specifically using the Support Vector Machine algorithms and neural networks. The tests were carried out on several distinct situations, followed by validation and verification tests on the devices designed and developed within the project (MIDA, Monitoil).
\end{abstract}

\section{Introduction}

A lot of methods in the fault (error) diagnosis literature are based on linear or accurate methodologies, or with a high dose accuracy. It is known that industrial processes are often difficult to model. These are complex and not precisely known; noise and uncertain, error-prone sensors corrupt measurements. Therefore, some researchers have perceived artificial neural networks as an alternative way to represent knowledge about malfunctions, but also to find ways to solve technical problems with infinitesimal errors, but sometimes also abruptly influenced by errors approach, or design. Neural networks can filter noise and disturbance; they can provide a stable diagnostic, failures without traditional types of models, extremely sensitive, and economic efficiency due to insignificant computing and design effort. Another desirable feature of neural networks is that exact patterns are not required to reach the decision stage [1], [2], [4], [5], [7], [8], [11]. In a typical operation, the process model can only be approximate and critical measurements may be capable of internally crunching functional relationships that represent processes, filtering noise, and managing correlations. Although there are many promising examples of neural network simulations in diagnosing errors in specific literature, real applications are still quite rare. There is a great need to carry out more detailed scientific investigations on the application of neural networks in real industrial installations to achieve full use of their attractive features.

Using a neural network with appropriate parameters and valid statistical architecture and weights, any continuous non-linear relationship can be approximated with arbitrary precision, a significant property that a relatively complicated but controllable algorithm as a mathematical process can be called by functions or specific applications in MATLAB, or dedicated software.

Neural networks are composed of simple elements that work in parallel. The biological nervous systems inspire these elements. As in nature, the network function is primarily determined by the connections between the elements. We can train a neural network to perform a specific function by adjusting the values of the connections (weights) between the elements, [3], [6]. Typically, conventional neural networks are adjusted, or trained, so that a particular entry leads to a specific target output. Such a situation is presented in Fig. 1.

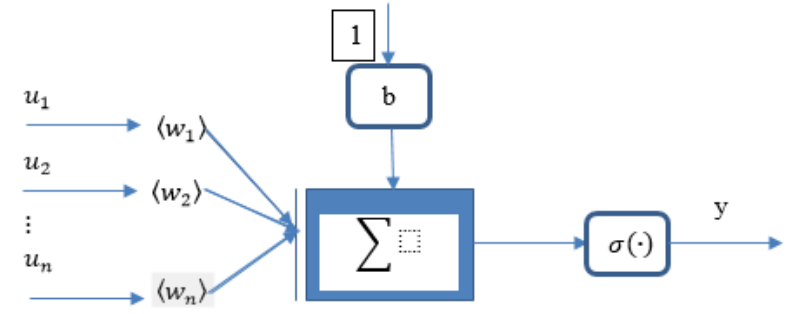

Fig. 1. Neuron scheme with $n$ inputs $\left(u_{i}\right), w_{i}$-weighted, $\operatorname{biased}(\mathrm{b}), \sigma(\cdot)$ is the non-linear activation function and one output (y)

Here, the neural network is adjusted, based on a comparison between output and target, until the network output matches the target. Typically, many such input/ target pairs are used to train a network, this type of neural network is called a supervised learning network. The classical mathematical model of such a neural network is described by the following equation, [1]:

$$
y=\sigma\left(\sum_{i=1}^{n} \omega_{i} u_{i}+b\right)
$$

* Corresponding author: ggrebenisan@gmail.com 
where $u_{i}$ are the system inputs, $w_{i}$ - the weights coefficients, b-the bias(treshold), $\sigma(\cdot)$ is the non-linear activation function and the output y. In Fig. 2. a singlelayer neural network, with $\mathrm{S}$ neurons, is presented. Note that each of the network inputs is connected to each of the network's neurons, for this reason the weight matrix now has $\mathrm{S}$ rows. The layer includes the $W_{i}, i=1 \ldots n$-weight's matrix, the summers, the bias vector, $b_{j}, j=1 \ldots s$, the transfer functions, $\sigma(\cdot)$, and the output vector, $y_{j}, j=$ $1 \ldots s$. Each element of the input vector $u_{i}$ is connected to each neuron through the weight matrix, $W_{i}, i=1 \ldots n$. Each neuron has a bias vector, $b_{j}, j=1 \ldots s$, a summer, a transfer function, $\sigma(\cdot)$, and an output vector, $y_{j}=$ $\sigma\left(W \cdot u_{i}+b_{j}\right), j=1 \ldots s$.

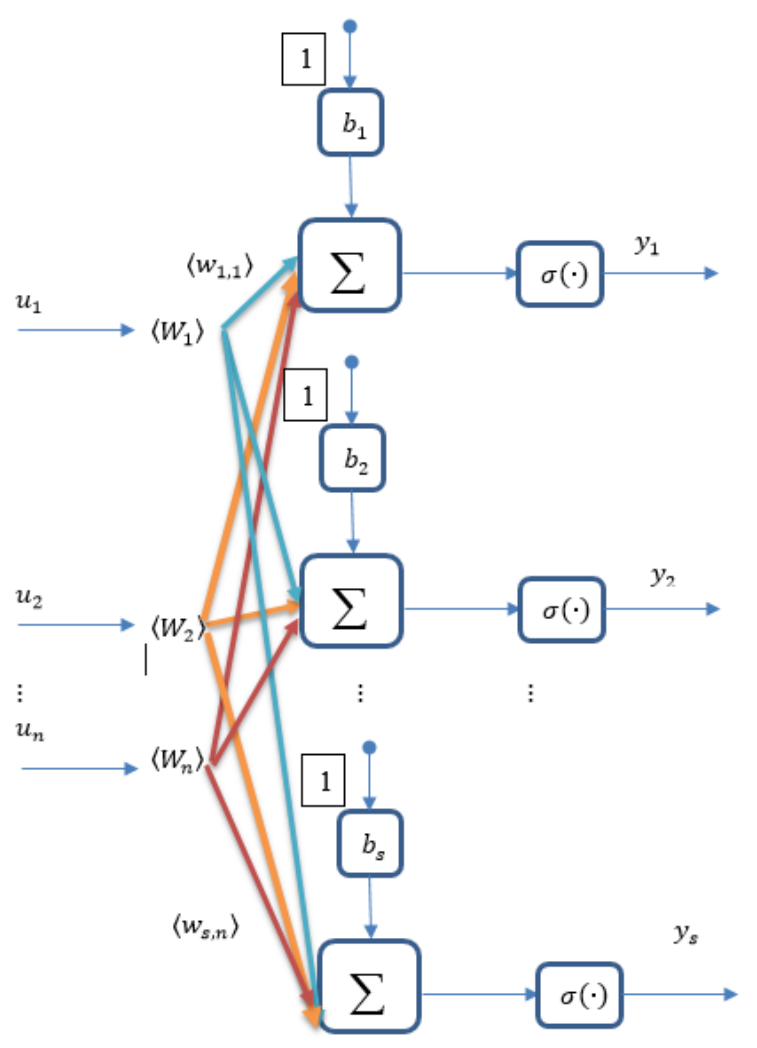

Fig. 2. A single layer network, with $\mathrm{n}$ inputs and $\mathrm{S}$ neurons

Not always the inputs vector size is similar to the number of neurons, it is quite no common to be equality between these characteristics. Also, not all neurons in a layer may have the same transfer function. In this case, it is common to define a single (composite) layer of neurons that have different transfer functions by combining two or more networks in parallel, depending on the number of different transfer functions. Thus the neural networks could have the same inputs and each network would create their own outputs. The weight matrix, W, trough which the inputs vector enter to the network is:

$$
W=\left[\begin{array}{cccc}
w_{1,1} & w_{1,2} & \cdots & w_{1, n} \\
w_{2,1} & w_{2,2} & \cdots & w_{2, n} \\
& \vdots & & \\
w_{S, 1} & w_{S, 2} & \cdots & w_{S, n}
\end{array}\right]
$$

Now we consider a multilayer neural network(Fig. 3.), [2]. Each layer has its own weighting matrix, its own bias vector, a vector with $\mathrm{n}$ inputs, and a vector of outputs, $y$ (the layers are identified, symbolically, by the superscript, which indicates its number, eg the weight matrix, in layer 3 is $W^{3}$ ). Thus, the output of the neural network is given by the relationship:

$$
y^{3}=\sigma^{3}\left(W^{3} \cdot \sigma^{2}\left(W^{2} \cdot \sigma^{1}\left(W^{1} \cdot u_{i}+b^{1}\right)+b^{2}\right)+b^{3}\right)
$$

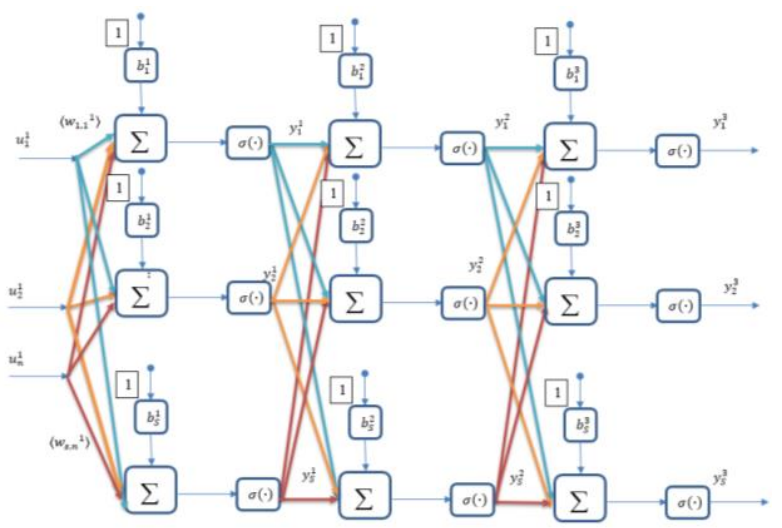

Fig. 3. The 3-layer neural network, with $\mathrm{n}$ inputs and $\mathrm{S}$ neurons, [9]

Some definitions shall be presented in order to use a unified terminology, [8]:

- The fault is an unaccepted deviation of at least one characteristic property or system variable from that characteristic of the acceptable / standard / standard behavior of the system.

- The failure is a permanent interruption of the system's ability to perform a function under certain operating conditions.

- The fault detection is an identification of the defects present in the system and the time required to detect them.

- Isolation of the fault is an identification of the type, location and time of detection of an error / malfunction. Follow fault detection.

- Identifying defects is a determination of behavioral deviation, size, and variance over time of a malfunction. The isolation of the fault follows.

- The fault diagnosis is a determination of the type, size, location, and detection time of a malfunction. Follow fault detection. Includes both the isolation of defects as well their identification. The main objective of fault diagnosis is to detect defects in each subsystem and their causes early enough so as to avoid global system failure and provide information about their size and sources. 
Even if the elements used in the current industrial systems are of high reliability, there are always failures of the components of the technological components and of the automatic devices as well as faults due to the mistakes of the operator. According to some specialists, the degradation states, which occur frequently during the maintenance of the technological components, are quite natural. They cause significant and long-term disturbances during the manufacturing process, which leads to a decrease in productivity and can sometimes result in complete, definitive damage to these systems. In these cases, economic losses are very high. Some malfunctions may lead to reactions that may result in environmental hazards or damage to production facilities. They can also be dangerous to human life. Identifying the failure state is usually a delicate problem, especially in the case of complex automated systems. The human operator may often be overwhelmed in short periods of time (such as accidents or successive chemical reactions). These systems are irremediably affected by the lack of a prompt decision taken by the human operator, or by the effects of delayed decisions, and in the absence of correct decisions, systems can evolve into defects or physically irreparable states. Problems of diagnosis and process protection are constantly developing. The importance of this problem increases with economic growth, with the degree of automation and justifies the development of maintenance and technological monitoring compartments. Therefore, computational systems that allow either operators to perform a correct diagnosis in the event of an alarm or fault detected or to establish a rational diagnosis in an automatic mode, which is essential.

Compared to diagnostic operations performed by an operator, the automation of diagnostic operations makes it possible to shorten the response times significantly, and to narrow the time elapsed between the time of identification and complete isolation of the malfunctions. This has the effect of increasing the reliability, the economic efficiency of protected systems and the development of automation in the field, [8].

\section{Databases used for classification using neural network}

Data on the condition of the oil parameters used in the hydraulic system for lubricating machine tools have been collected for six months. The data are matrixed, with 258648 rows (observations) and 21 columns (parameters). The parameters are presented in Table 1, [12].

Developing and testing prototype variants of experimental data monitoring and collection systems, analyzing continuous signals to identify the relevant features of the investigated parameters, and determining the "useful" data collection space to be able to provide an estimate accurate, or as close as possible, were designed to create the conditions and the mathematical environment that are conducive to the development of Support Vector Machines and Classification and Parameters Estimation (using neural network based modeling) algorithms suitable for reading the signal characteristics on the basis of "learning".
Table 1 The parameters collected, [12]

\begin{tabular}{|l|l|}
\hline id & measure identifier \\
\hline postgresq1_timestamp & writing time \\
\hline time & writing time \\
\hline raw_data & $\begin{array}{l}\text { Maybe neglected, it is not } \\
\text { important (data before process } \\
\text { starts) }\end{array}$ \\
\hline icm_isocode & $\begin{array}{l}\text { ISO code 4406 (icm_iso4/ } \\
\text { icm_iso6/ icm_iso14) }\end{array}$ \\
\hline icm_rh & water saturation\% \\
\hline icm_flow & $\begin{array}{l}\text { oil flow through particle } \\
\text { counter }\end{array}$ \\
\hline icm_temp & $\begin{array}{l}\text { particle counter electronic } \\
\text { temperature }\end{array}$ \\
\hline icm_iso4 & $\begin{array}{l}\text { ISO } 4406 \text { code class particles } \\
>4 \mu m\end{array}$ \\
\hline icm_iso6 & $\begin{array}{l}\text { ISO } 4406 \text { code class particles } \\
>6\end{array}$ \\
\hline icm_iso14 & $\begin{array}{l}\text { ISO } 4406 \text { code class particles } \\
>14 \mu \mathrm{m}\end{array}$ \\
\hline icm_pc4 & number of particles $>4 \mu \mathrm{m}$ \\
\hline icm_pc6 & number of particles $>6$ m \\
\hline icm_pc14 & number of particles $>14 \mu \mathrm{m}$ \\
\hline fps_vcst & viscosity [cSt] \\
\hline fps_v & viscosity [cP] \\
\hline fps_density & density \\
\hline fps_dielectric & dielectric constant \\
\hline fps_temp & oil temperature \\
\hline oh_temp & Oilhealth electronic temperature \\
\hline oh_parama & $\begin{array}{l}\text { Oilhealth paramA (not } \\
\text { important) }\end{array}$ \\
\hline oh_paramb & $\begin{array}{l}\text { Oilhealth paramB (not } \\
\text { important) }\end{array}$ \\
\hline oh_paramc & Oilhealth (not important) \\
\hline oh_od & Index of degradation OD\% \\
\hline Date & day of analysis \\
\hline time & hour/time of analysis \\
\hline & \\
\hline
\end{tabular}

Table 2. Data collected- the first 30 from 258648 rows and all columns of the database, are showed, [12]

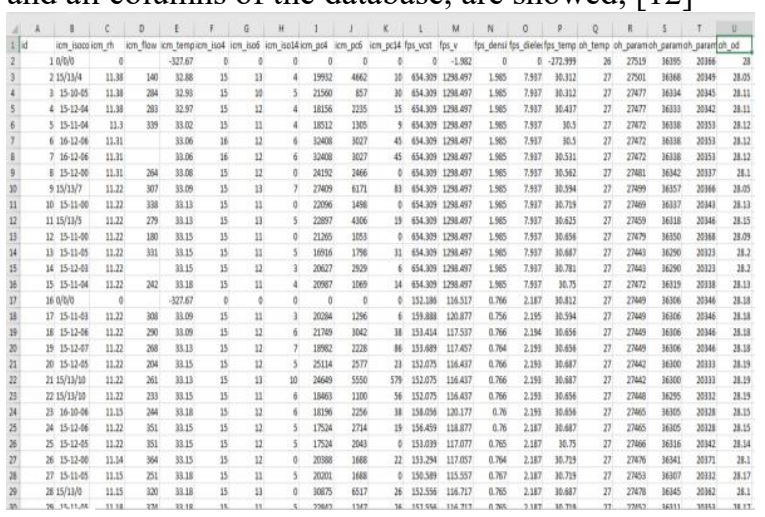

Experiments and in-situ trials have been carried out considering the basis of data usage using on-line analysis, which means efficiency and reproducibility, [10].

\section{Results and validation discussion}

Testing, training, and validation of the collected data set ( $\mathrm{N}$ observations) were possible through mathematical and experimental statistical operations, by generating/ separating $\mathrm{K}$ different time frames (each consisting of $\mathrm{N}$ / 
$\mathrm{K}$ data) and analyzing the signal characteristics on each of these frames (which have the advantage of being composed of data that is not repeated and therefore not linearly dependent). Alternatively, the signal was divided into a larger number of frames that overlap partially (at the limit, NK-1 frames, provided that the first contains the data between $1 \ldots \mathrm{K}$, the second zone $2 \ldots \mathrm{K}+1$, etc.). In this way, the data is partially superimposed on a given order, but the advantage is that the data set becomes a time series of parameters in a smoother regression and therefore easier to extrapolate. The data set consists of 5431608 observations collected every minute from a work machine. The data refer to the analyzes made on the manufacturing line on the following parameters: density, viscosity, dielectric, water, oil degradation, temperature, ISO4, ISO6, ISO14, with obvious importance for the oil condition, but also for the operation of the industrial equipment being tested (partialy showed on Table 2, and graphical represented in plot figure, Fig. 4.) .

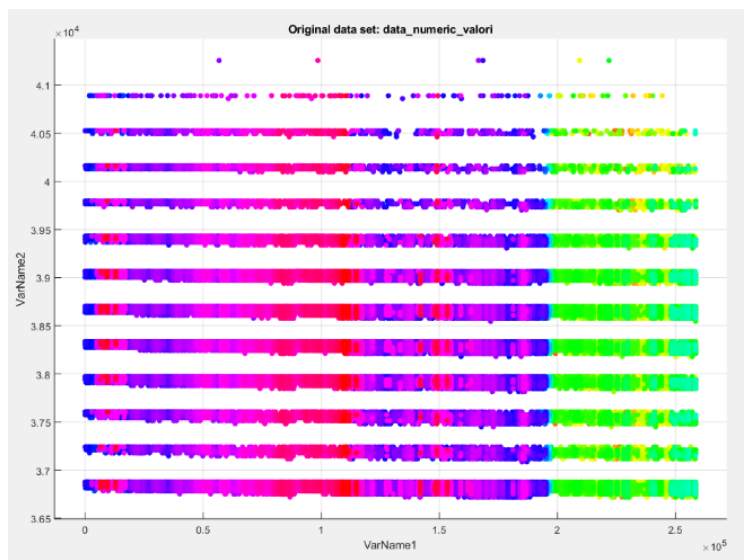

Fig. 4. Original data set (the all 5431608 parameters)

Once the dataset was received, the project coordination team, conducted data analysis, customized software development, and data set configuration to "clean up" the missing data set. Later, the "learning" of the machine was performed using the Support Vector Machine for a class (Fig. 5.).

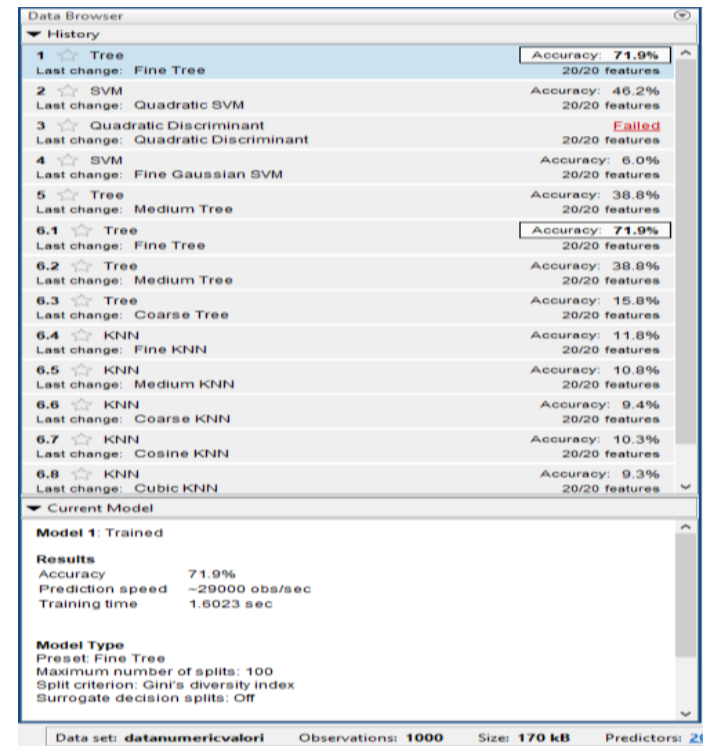

Fig. 5. The training phase, using Support Vector Machine
At the end of experimentation on this first set of data, it was decided to perform the training phase using 50 minute superimposed frames (the first frame consists of the data collected between minute 1 and minute 50, the second frame consists of data collected between minute 2 and minute 51, and so on). With the data in each frame the best fit is based on a model based on the ARIMA model whose parameters are the characteristics used for the training. The training phase, including a cross-validation part, generated SVM machine, resulting in machine training in a class with an accuracy of more than $70 \%$ (Fig. 5.). Support Vector Machine is used for training on the computing units available, capable of analyzing a 50 minute observation sample and performing the test using SVM in less than one second by the computing processor (Artificial intelligence).
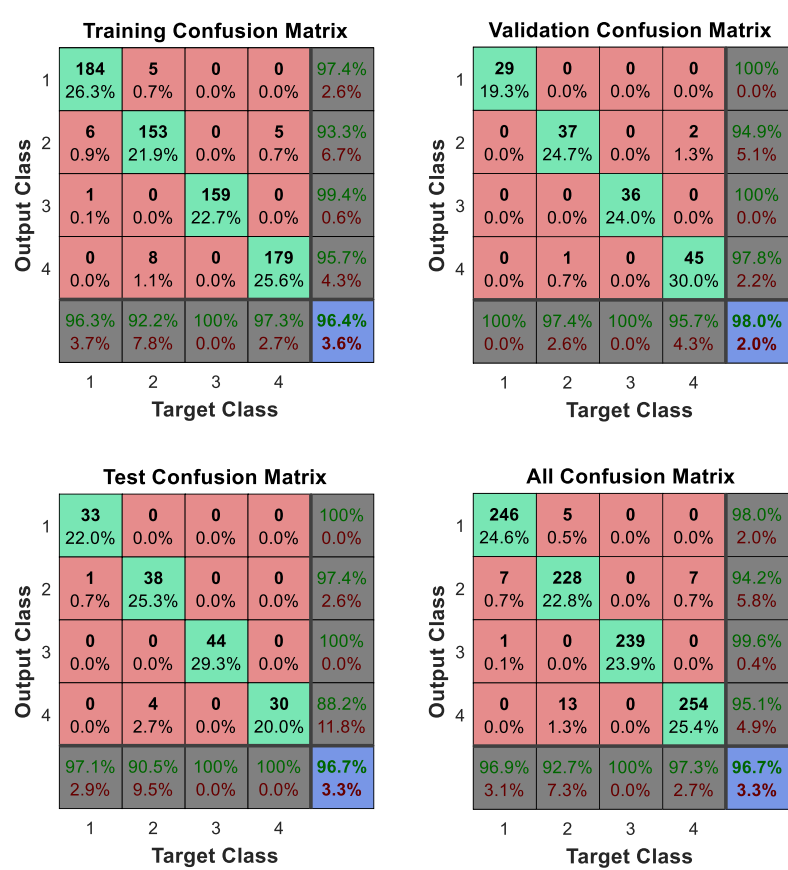

Fig. 6. Training Confusion Matrix, Testing Confusion Matrix, Validation Confusion Matrix, the summary of Confusion Matrix

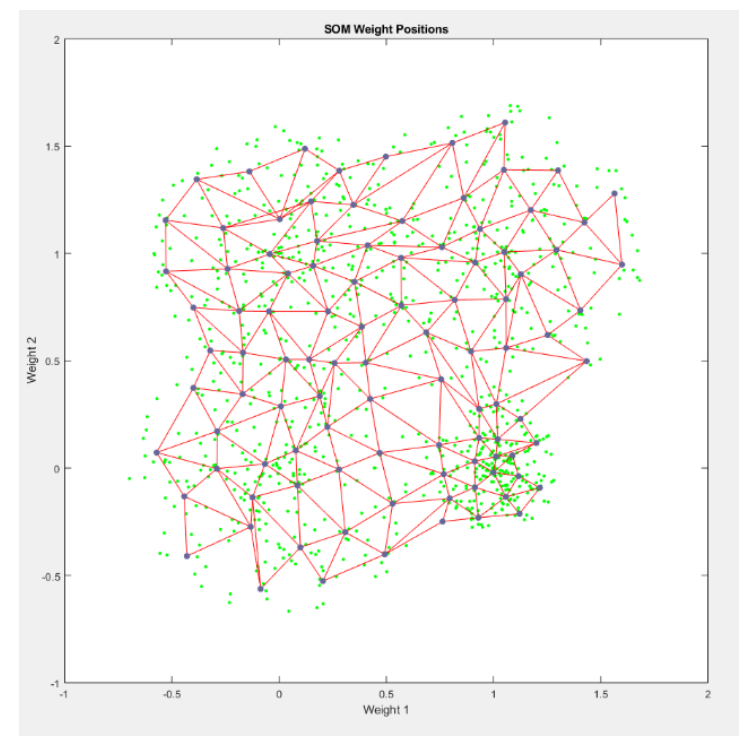

Fig. 7. The regression learner for selected data 

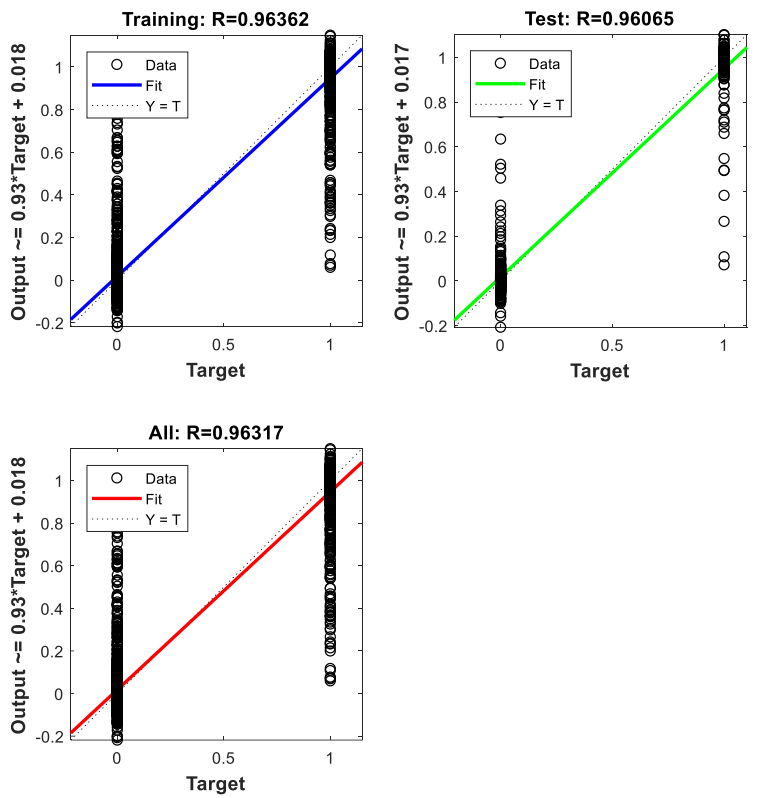

Fig. 8. The regression plot (SVM)

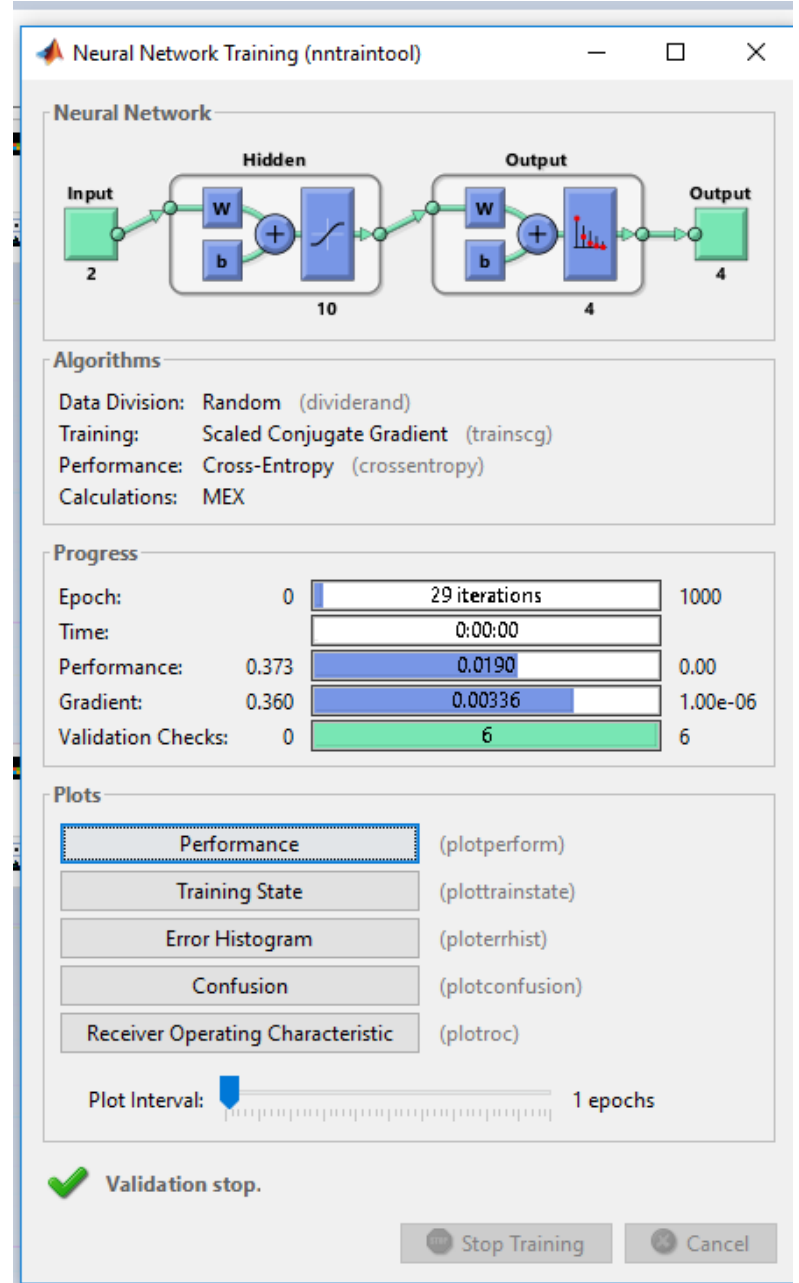

Fig. 9. The neural network training results and coordinates

The results of the training using the neural network algorithm are presented in Fig. 9. Here you can notice the architecture of the network as well as the numerical parameters of the network. In Fig. 10, respectively in Fig. 11 . The results of the tree classification are represented graphically in two variants: complex tree, and simplified tree, respectively, [9], [10].

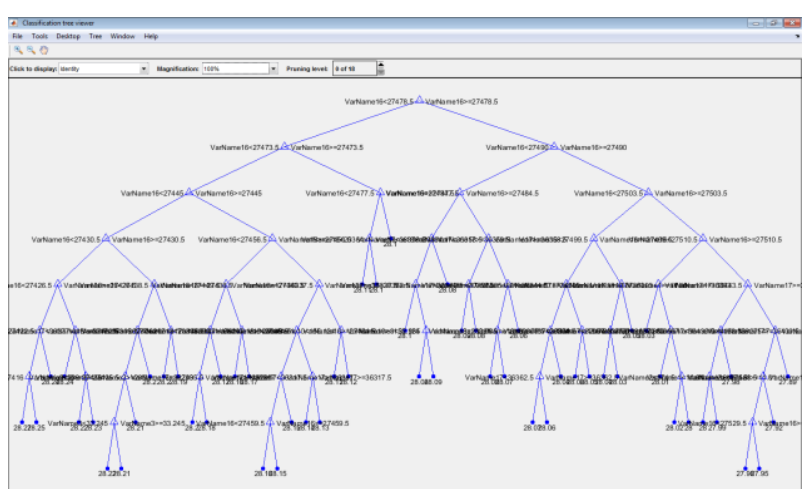

Fig. 10. Complex Tree

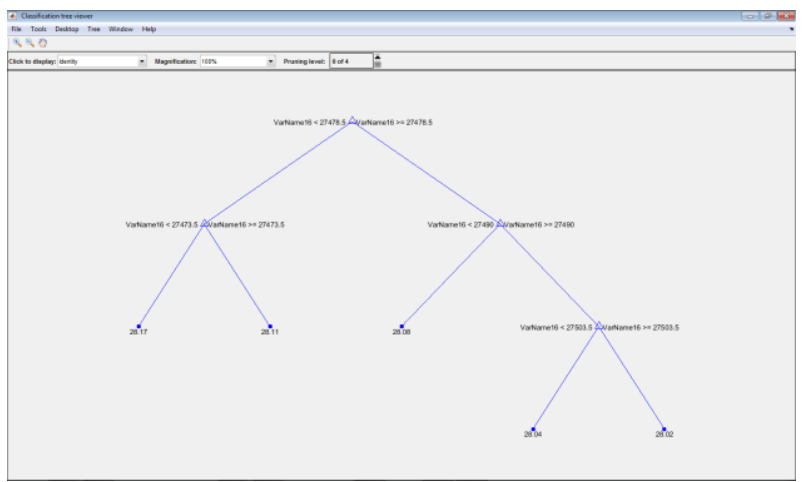

Fig. 11. Simple Tree

In Fig. 12. the distribution in two dimensions of the oil status parameters analyzed in the research project is represented, ie the influence of the content of hydrological contaminants and the impurities on the viscosity of the lubricant. Classification accuracy was $63 \%$ for a SVMComplex Tree algorithm.

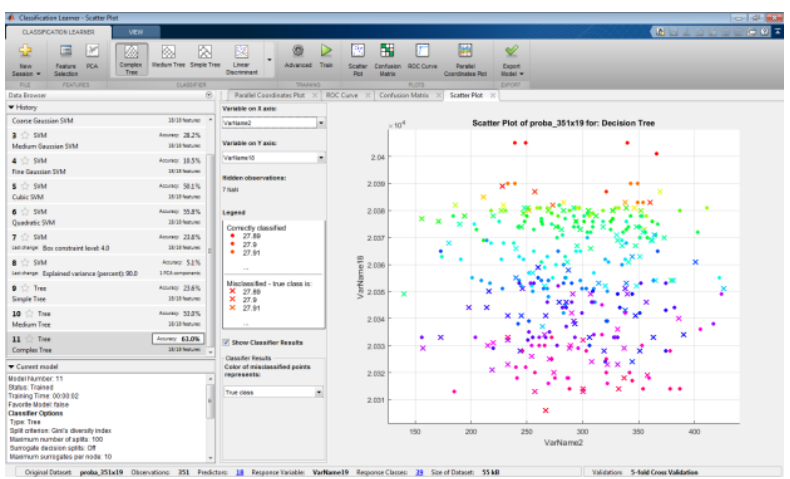

Fig. 12. Scatter Plot

Perhaps a more eloquent and deeper exposure to the behavior of the classifier is revealed by the so-called matrix of confusion. The i, jth element of this matrix represents the number of $\omega_{i}$ samples in the validation set to which the $\omega_{\mathrm{j}}$ class is assigned. The confusion matrix is graphically presented in Fig. 6 and Fig. 13. 


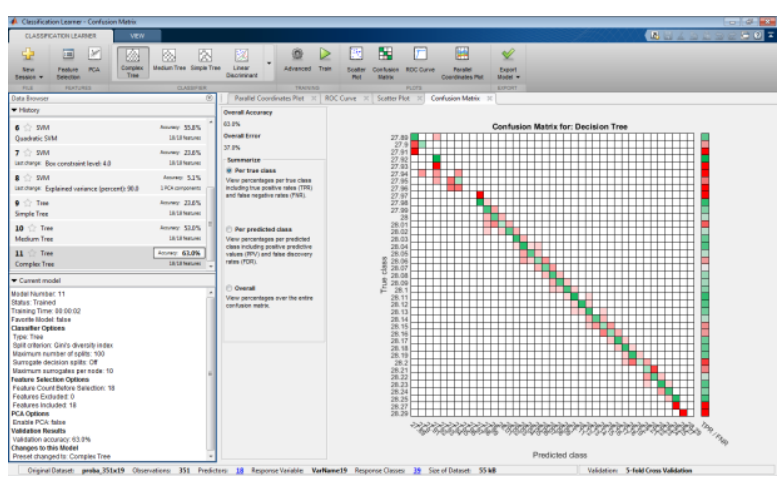

Fig. 13. The Confusion Matrix

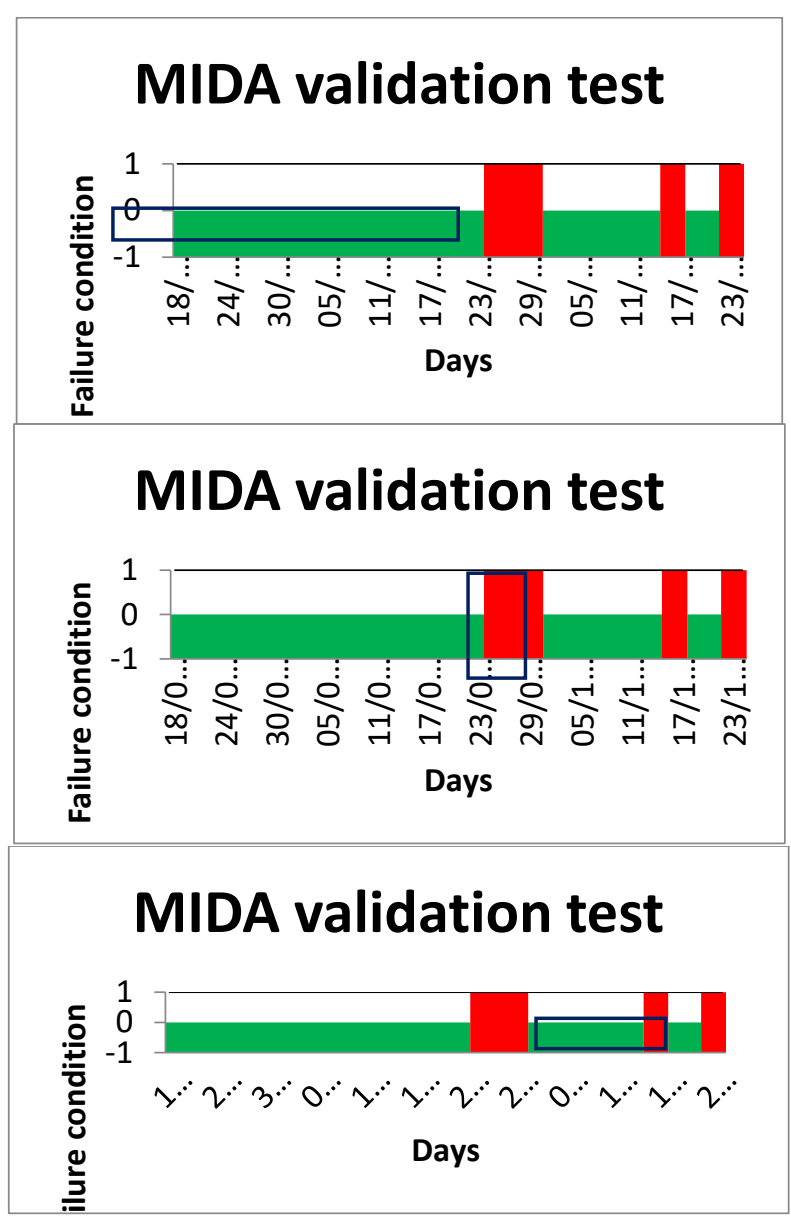

Fig. 15. The validation tests, results on MIDA application

\section{Conclusion}

The algorithm on which the system operates is stable, not influenced by variations in operating parameters. The system has an extremely simple operating principle and does not require additional staff training. The system works on-line: the speed of response to errors in measurement is very high. Alarms are generated to exceed the preset (learned) values of the parameters. The system can be customized for any industrial equipment. The system is adaptable as it can be "taught" to determine the errors that occurred during the monitoring of any process. The system is safe, with no possibility of interference during data collection or transmission of collected data. The system is replicable. System improvements are possible: as regards the computing system (more efficient processor); as regards the speed of data acquisition; regarding the speed of data transmission. Application of the system to other types of equipment in other fields is always possible (in aviation, auto, etc.). The system can be certified.

\section{Acknowledgment}

This work is supported by Machine Intelligence for Diagnosis Automation (MIDA), Research Program, National Program PN II, ERA MANUNET: NR 13081221/13.08.2013

\section{References}

1. K. Patan- Artificial Neural Networks for the Modelling and Fault Diagnosis of Technical Processes, LNCIS 377, Springer-Verlag Berlin Heidelberg 2008.

2. H. Demuth, M. Beale-Neural Network Toolbox For Use with MATLAB ${ }^{\circledR}$, The MathWorks, Inc., 2014.

3. M. T. Hagan, H. B. Demuth, M. H. Beale, O. De Jesús- Neural Network Design, Second Edition, 2014, ebook.

4. P. M. Frank, B. Koppen-Seliger New Developments Using AI in Fault Diagnosis, Engng. Applic. Artif Intel. Vol. 10, No. 1, pp. 3-14, 1997 (C) 1997 Elsevier Science Ltd.

5. H.N. Kolvo, Artificial Neural Networks In Fault Diagnosis And Control, Control Eng. Practice, Vol. 2, No. 1, pp 89-101, 1994, Printed in Great Britain.

6. J. R. Rabunal and J. Dorrado Artificial Neural Networks In Real-Life Applications, Idea Group Publishing, 2006, London.

7. L. H. Chiang, E. L. Russell and R. D. Braatz, Fault detection and diagnosis in industrial systems, Springer-Verlag London Limited, 2002.

8. J. Korbicz : J.M. Koscielny - Z. Kowalczuk : W. Cholewa, Fault Diagnosis -Models, Artificial Intelligence, Applications, Springer-Verlag Berlin Heidelberg GmbH, 2004.

9. B. Lei, D. de Ridder, D. M. J. Tax, F. van der Heijden, G. Xu, M. Feng, Y. Zou - Classification, Parameter Estimation and State Estimation An Engineering Approach Using MATLAB, Second Edition, 2017 JohnWiley \& Sons, Ltd.

10. A. Gelman and J. Hill, Data Analysis Using Regression and Multilevel/Hierarchical Models, Cambridge University Press, 2006.

11. J. Korbicz, M. Mrugalski: Confidence estimation of GMDH neural networks and its application in fault detection systems, International Journal of Systems Science (2007) DOI: 10.1080/00207720701847745

12. G. Grebenişan, S. Bogdan, N. Salem: The lubricants parameters monitoring and data collecting, Annual Session Of Scientific Papers, "IMT ORADEA 2018”, Volume XXVII (XVII), ISBN 978-606-101537-5, Oradea, 2018 